\title{
RECORD-4 multicenter phase 2 trial of second-line everolimus in patients with metastatic renal cell carcinoma: Asian versus non-Asian population subanalysis
}

Lin Yang ${ }^{1 *}$, Anna Alyasova ${ }^{2}$, Dingwei Ye ${ }^{3}$, Antonia Ridolfi ${ }^{4}$ Luca Dezzani ${ }^{5}$ and Robert J. Motzer ${ }^{6}$

\begin{abstract}
Background: RECORD-4 assessed everolimus in patients with metastatic renal cell carcinoma (mRCC) who progressed after 1 prior anti-vascular endothelial growth factor (VEGF) or cytokine and reinforced the clinical benefit of second-line everolimus. Because of the high percentage of patients from China enrolled in RECORD-4 (41\%) and some reported differences in responses to certain targeted agents between Chinese and Western patients, this subanalysis evaluated outcomes in Asian versus non-Asian patients.

Methods: RECORD-4 enrolled patients with clear cell mRCC into 3 cohorts based on prior first-line therapy: sunitinib, other anti-VEGF (sorafenib, bevacizumab, pazopanib, other), or cytokines. Patients received everolimus $10 \mathrm{mg} / \mathrm{d}$ until progression of disease (RECIST, v1.0) or intolerance. Primary end point was progression-free survival per investigator review. Data cutoff was Sept 1, 2014.

Results: Among Asian ( $n=55)$ versus non-Asian $(n=79)$ patients, $98 \%$ versus $84 \%$ had good/intermediate MSKCC prognosis; $73 \%$ versus $65 \%$ were men, and $85 \%$ versus $73 \%$ were $<65$ years of age. All (100\%) Asian patients were of Chinese ethnicity. Median duration of exposure was $5.5 \mathrm{mo}$ for Asian and $6.0 \mathrm{mo}$ for non-Asian patients. Among Asian versus non-Asian patients, median progression-free survival (months) was 7.4 versus 7.8 overall, 7.4 versus 4.0 with prior sunitinib, and 5.7 versus 9.2 with prior other anti-VEGFs. Clinical benefit rate was similar between populations: $74.5 \%$ (95\% Cl 61.0-85.3) for Asian patients and 74.7\% (95\% Cl 63.6-83.8) for non-Asian patients. Most patients achieved stable disease as best overall response (Asian, 63.6\%; non-Asian, 69.6\%). Overall rate of grade 3/4 adverse events appeared similar for Asian (58\%) and non-Asian patients (54\%).
\end{abstract}

Conclusions: This RECORD-4 subanalysis demonstrated comparable efficacy and adverse event profiles of secondline everolimus in Asian and non-Asian patients. Efficacy and safety outcomes by prior therapy should be interpreted with caution because of small patient numbers in some subpopulations.

Trial registration: Everolimus as Second-line Therapy in Metastatic Renal Cell.

Carcinoma (RECORD-4); ClinicalTrials.gov identifier: NCT01491672. Registration date: December 14, 2011.

Keywords: Asians, Everolimus, Ethnicity, Clear-cell metastatic renal cell carcinoma, Sequence

\footnotetext{
* Correspondence: lyang69@sina.com

${ }^{1}$ National Cancer Center/Cancer Hospital, Chinese Academy of Medical

Sciences and Peking Union Medical College, 17 Panjiayuannanli, Chaoyang

District, Beijing, China

Full list of author information is available at the end of the article
} 


\section{Background}

Everolimus, a mammalian target of rapamycin inhibitor, is a second-line treatment option for patients with metastatic renal cell carcinoma (mRCC) refractory to vascular endothelial growth factor (VEGF) receptor inhibitors [1, 2]. Approval of everolimus was based on results of the phase 3 RECORD-1 study of patients who had previously received sunitinib, sorafenib, or both [3]. The phase 2 RECORD-4 study was subsequently designed to assess everolimus as a purely second-line therapy in patients with mRCC [4]. Because $41 \%$ of RECORD-4 patients were from China, and some differences in responses to certain targeted agents between Chinese and Western patients have been reported [5], this subanalysis evaluated outcomes in Asian patients compared with non-Asian patients.

\section{Methods}

The RECORD-4 primary analysis has been published [4]. Briefly, 134 adult patients with confirmed clear cell mRCC (RECIST v1.0) and a Karnofsky performance status of $\geq 70 \%$ were enrolled into 1 of 3 cohorts based on their previous first-line therapy: sunitinib, other antiVEGF agents (sorafenib, bevacizumab, pazopanib, tivozanib, and axitinib), or cytokines. Patients received everolimus $10 \mathrm{mg} / \mathrm{d}$ until disease progression, unacceptable toxicity, treatment discontinuation, or death. Dose reduction to $5 \mathrm{mg} / \mathrm{d}$ was permitted to manage adverse events (AEs). The primary end point was progressionfree survival (PFS) per local radiologic assessment. Median PFS was 7.8 months (95\% confidence interval [CI] 5.7-11.0) in the overall population, 5.7 months (95\% CI 3.7-11.3) in the first-line sunitinib cohort, 7.8 months (95\% CI 5.7-11.0) in the first-line other anti-VEGF therapy cohort, and 12.9 months (95\% CI 2.6-not estimable $[\mathrm{NE}])$ in the first-line cytokine-based therapy cohort.

\section{Results \\ Patients}

In the Asian $(n=55)$ and non-Asian $(n=79)$ populations, respectively, $98 \%$ and $84 \%$ had good/intermediate Memorial Sloan Kettering Cancer Center (MSKCC) prognosis, $73 \%$ and $65 \%$ were men, and $85 \%$ and $73 \%$ were $<65$ years of age (Additional file 1: Table S1). Median duration of exposure was 5.5 and 6.0 months for Asian and non-Asian patients, respectively.

\section{Efficacy}

Median PFS was 7.4 months (95\% CI 5.5-11.0) for Asian patients $(n=55)$ and 7.8 months (95\% CI 5.3-12.9) for non-Asian patients $(n=79)$ (Fig. 1a). In the first-line sunitinib cohort, median PFS was 7.4 months (95\% CI 3.7-12.8) in the Asian population $(n=29)$ and 4.0 months (95\% CI 2.5-12.9) in the non-Asian population $(n=29)$ (Fig. 1b). In the first-line other anti-VEGF agents cohort, median PFS was 5.7 months (95\% CI 3.6$11.0)$ in the Asian population $(n=21)$ and 9.2 months (95\% CI 5.5-18.0) in the non-Asian population $(n=41)$ (Fig. 1c). In the first-line cytokines cohort, median PFS was 16.5 months (95\% CI 1.9-NE) in the Asian population $(n=5)$ and 12.9 months (95\% CI 2.6-NE) in the non-Asian population $(n=9)$ (Fig. 1d). Best overall response was stable disease for $64 \%$ and $70 \%$ of Asian $(n=35)$ and non-Asian patients $(n=55)$, respectively (Additional file 2: Table S2). Clinical benefit rate (complete response + partial response + stable disease) was $75 \%$ (95\% CI $61.0-85.3)$ for Asian patients $(n=41)$ and $75 \%$ (95\% CI 63.6-83.8) for non-Asian patients $(n=$ 51).

\section{Adverse event profile}

The overall rate of grade 3 and 4 AEs (irrespective of relationship to study drug) was $58 \%$ and $54 \%$ for Asian and non-Asian patients, respectively (Additional file 3: Table S3). The most common grade 3 and 4 AEs were anemia (7\%), decreased hemoglobin level (6\%), hypertriglyceridemia (6\%), mouth ulceration (6\%), proteinuria (6\%), respiratory failure (6\%), and stomatitis (5\%) among Asian patients and anemia (17\%), hyperglycemia (5\%), and stomatitis (5\%) among non-Asian patients. In the Asian and non-Asian populations, respectively, 22 (40\%) and $35(45 \%)$ patients required dose adjustment or study drug interruption to manage AEs. Eleven patients (20\%) in the Asian population and 13 patients (17\%) in the non-Asian population discontinued treatment because of AEs. Causes of on-treatment deaths in the Asian population were disease progression $(n=3)$, respiratory failure $(n=2)$, and multiorgan failure $(n=1)$ and in the nonAsian population were multiorgan failure $(n=2)$, cardiopulmonary failure $(n=1)$, disease progression $(n=1)$, sepsis $(n=1)$, sudden death $(n=1)$, and unknown cause $(n=1)$.

\section{Discussion}

There have been some reported differences in responses to certain targeted agents between Chinese and Western patients with RCC [5]. However, our findings support the comparable efficacy of everolimus in Asian and nonAsian patients with RCC. In this RECORD-4 subanalysis of second-line everolimus, median PFS was 7.4 and 7.8 months in Asian and non-Asian patients, respectively, and the clinical benefit rate was $75 \%$ in both patient populations. These findings suggest that secondline everolimus has comparable efficacy in Asian and non-Asian patients with RCC. These results are supported by a phase $1 \mathrm{~b}$ study of everolimus in 64 Chinese mRCC patients who were intolerant to, or progressed on, prior VEGFR-TKI therapy, in which median PFS was 6.9 months and the clinical benefit rate was 66\% [6]. 


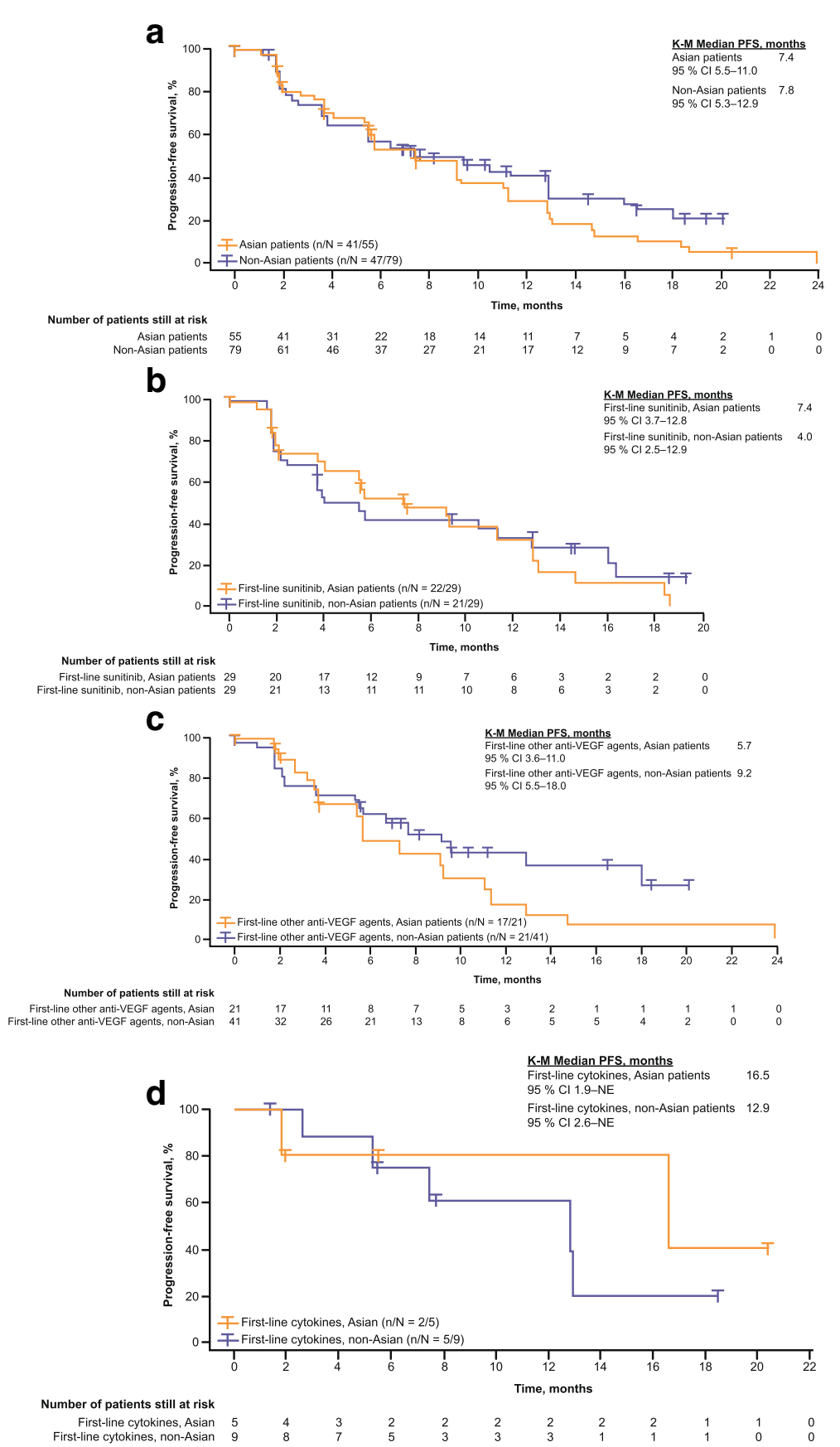

Fig. 1 Progression-free survival (PFS) in the Asian and non-Asian populations (a), and by first-line treatment cohorts in the Asian and non-Asian populations for (b) sunitinib, (c) other anti-VEGF agents, and (d) cytokines. Cl=confidence interval; K-M=Kaplan-Meier; NE=not estimable; PFS=progression-free survival; VEGF=vascular endothelial growth factor

Median PFS was comparatively shorter (4.9 months) in the pivotal phase 3 RECORD-1 trial of everolimus in VEGFR-TKI pretreated mRCC patients from centers across Australia, Canada, Europe, the USA, and Japan [3]. However, patients in RECORD-1 were more heavily pretreated, having received a median of 2 prior antineoplastic therapies, and had a poorer risk profile per MSKCC criteria, which may negatively affect outcomes in RECORD-1 compared with RECORD-4 [3, 4].

Five targeted drugs (pazopanib, everolimus, axitinib, sorafenib, and sunitinib) are currently approved for the treatment of advanced RCC in China, and everolimus and axitinib carry the highest level of evidence for second-line treatment after failure of first-line TKI in 
Chinese and Asia consensus treatment guidelines [7-9]. Thus, results of our study are important to physicians who treat these populations of patients, and support the use of everolimus following progression on first-line TKI therapy in Asian patients with mRCC. It is important that outcomes by prior therapy should be interpreted with caution because of the small patient numbers in some subpopulations.

\section{Conclusions}

In conclusion, second-line everolimus had comparable efficacy and safety in Asian and non-Asian patients with mRCC.

\section{Additional files}

Additional files 1: Table S1 Baseline demographics and disease characteristics of Asian and non-Asian patients in the overall population and in the first-line therapy cohorts. (DOCX $15 \mathrm{~kb}$ )

Additional files 2: Table S2 Tumor responses (RECIST v1.0) of Asian and non-Asian patients in the overall population and in the first-line therapy cohorts. (DOCX $13 \mathrm{~kb}$ )

Additional files 3: Table S3 Grade 3 and 4 adverse events reported by Asian and non-Asian patients in the overall population and in the firstline therapy cohorts. (DOCX $13 \mathrm{~kb}$ )

\section{Abbreviations}

AEs: Adverse events; mRCC: Metastatic renal cell carcinoma; MSKCC: Memorial Sloan Kettering Cancer Center; PFS: Progression-free survival; RECIST: Response Evaluation Criteria In Solid Tumors; VEGF: Vascular endothelial growth factor

\section{Acknowledgments}

We thank the following investigators for their contributions to the study: Hanzhong Li, Boris Alekseev, Liping Xie, Galina Kurteva, Ruben Kowalyszyn, Oleg Karyakin, Yeni Neron, and Thomas Cosgriff. We also thank LaTonya Collins, Thomas Brechenmacher, Chinjune Lin, and Liza Morgan for their contributions to the study design, data analysis, and statistical analysis. Patients treated at Memorial Sloan Kettering Cancer Center were supported in part by Memorial Sloan Kettering Cancer Center Support Grant/Core Grant (P30 CA008748).

\section{Funding}

This study was funded by Novartis Pharmaceuticals Corp. Editorial assistance was provided by Cathy R. Winter, PhD (ApotheCom, Yardley, PA) and funded by Novartis Pharmaceuticals Corp. Patients treated at Memorial Sloan Kettering Cancer Center were supported in part by Memorial Sloan Kettering Cancer Center Support Grant/Core Grant (P30 CA008748). The funding body had no role in the design of the study and collection, analysis, and interpretation of data and in writing the manuscript.

\section{Availability of data and materials}

The datasets used and/or analysed during the current study are available from the corresponding author on reasonable request.

\section{Authors' contributions}

LY: conception and design, acquisition of data, drafting of the manuscript, critical revision of the manuscript, supervision; AA: acquisition of data, drafting of the manuscript, critical revision of the manuscript; DY: acquisition of data, drafting of the manuscript, critical revision of the manuscript; AR: analysis and interpretation of data, drafting of the manuscript, critical revision of the manuscript, statistical analysis, administrative, technical, or material support; LD: analysis and interpretation of data, drafting of the manuscript, critical revision of the manuscript, obtaining funding, administrative, technical, or material support; RJM: conception and design, acquisition of data, drafting of the manuscript, critical revision of the manuscript, supervision. All authors read and approved the final manuscript.

\section{Authors' information}

not applicable.

\section{Ethics approval and consent to participate}

The study was conducted in accordance with the International Conference on Harmonisation Good Clinical Practice guidelines, and approved by institutional review boards or independent ethics committees at each center. All patients provided written informed consent.

\section{Consent for publication}

not applicable.

\section{Competing interests}

AA received compensation from Novartis for research. AR and LD are employees of Novartis Pharmaceuticals Corp. RJM received compensation from Novartis, BMS, Pfizer, Genentech/Roche and Eisai for research and from Novartis, Pfizer, and Eisai for consulting. LY and DY have declared no competing interests.

\section{Publisher's Note}

Springer Nature remains neutral with regard to jurisdictional claims in published maps and institutional affiliations.

\section{Author details}

${ }^{1}$ National Cancer Center/Cancer Hospital, Chinese Academy of Medical Sciences and Peking Union Medical College, 17 Panjiayuannanli, Chaoyang District, Beijing, China. ${ }^{2}$ Prevoljskiy Region Medical Centre, llinskaua St, N, Novgorod 603109, Russia. ${ }^{3}$ Fudan University Shanghai Cancer Center, 220 Handan Rd, Yangpu, Shanghai, China. ${ }^{4}$ Novartis Pharma S.A.S, 2 Rue Lionel Terray, 92500 Rueil-Malmaison, France. ${ }^{5}$ Novartis Pharmaceuticals Corporation, 1 Health Plaza, East Hanover, NJ 07936, USA. ${ }^{6}$ Memorial Sloan Kettering Cancer Center, 1275 York Ave, New York, NY 10065, USA.

Received: 20 June 2017 Accepted: 5 February 2018

Published online: 17 February 2018

References

1. Escudier B, Porta C, Schmidinger M, et al. Renal cell carcinoma: ESMO clinical practice guidelines for diagnosis, treatment and follow-up. Ann Oncol. 2014;25(suppl 3):iii49-56.

2. National Comprehensive Cancer Network, Inc. NCCN Clinical Practice Guidelines in Oncology_Kidney Cancer [Version 3. 2016; cited 2016 June 1]. Available from: http://www.nccn.org/professionals/physician_gls/pdf/kidney. pdf.

3. Motzer RJ, Escudier B, Oudard S, et al. Phase 3 trial of everolimus for metastatic renal cell carcinoma: final results and analysis of prognostic factors. Cancer. 2010;116:4256-65.

4. Motzer RJ, Alyasova A, Ye D, et al. Phase 2 trial of second-line everolimus in patients with metastatic renal cell carcinoma (RECORD-4). Ann Oncol. 2016; 27(3):441-8

5. Tan X, Liu Y, Hou J, Cao G. Targeted therapies for renal cell carcinoma in Chinese patients: focus on everolimus. Onco Targets Ther. 2015:8:313-21.

6. Guo J, Huang Y, Zhang X. Safety and efficacy of everolimus in Chinese patients with metastatic renal cell carcinoma resistant to vascular endothelial growth factor receptor-tyrosine kinase inhibitor therapy: an open-label phase 1b study. BMC Cancer. 2013:13:136.

7. Zhou Q, Chen XY, Yang ZM, et al. The changing landscape of clinical trial and approval processes in China. Nat Rev Clin Oncol. 2017;14(9):577-83.

8. Guo J, Ma J, Sun Y, et al. Chinese guidelines on the management of renal cell carcinoma (2015 edition). Ann Transl Med. 2015;3(19):279.

9. National Comprehensive Cancer Network. NCCN Clinical Practice Guidelines in Oncology. Kidney Cancer. Asia Consensus Statements Version 32016 https://www.nccn.org/professionals/physician_gls/PDF/kidney-asia.pdf. Accessed 9 Feb 2018. 